\title{
Transport Phenomena of Inversion Layers in High Magnetic Fields
}

\author{
H. Obloh ${ }^{1}$, M. Dobers ${ }^{1}$, R. J. Haug ${ }^{1}$, K. v. Klitzing ${ }^{1}$ and D. Weiss ${ }^{2}$ \\ I Max-Planck-Institut für Festkörperforschung, Heisenbergstr. 1, D-7000 Stuttgart 80, Federal Republic of Germany \\ 2 Department of Physics E16, TU München, James-Franck-Str., D-8046 Garching, Federal Republic of Germany
}

\begin{abstract}
The discovery of the quantum Hall effect in 1980 has demonstrated the importance of two-dimensional electronic systems for application and fundamental research. In this paper a review of some transport phenomena in such systems in high magnetic fields is given.
\end{abstract}

\section{Introduction}

In $1980 \mathrm{v}$. Klitzing et al. [1] proposed a highly accurate method to determine the fine-structure constant $\alpha$ based on a new solid-state quantum effect - the quantum Hall resistance. This effect is connected with the behaviour of a disordered two-dimensional electron gas (2DEG) in high magnetic fields, but does not depend on device parameters. Experimentally, the quantized value of the Hall resistance $\varrho_{x y}=h /\left(e^{2} \cdot \mathrm{i}\right)$ (i: filling factor) has been found to be the same on silicon metaloxide-semiconductors ( $\mathrm{Si}$ MOSFETs) and GaAs- $\mathrm{Al}_{x} \mathrm{Ga}_{1-x}$ As heterostructures to within less than $0.2 \mathrm{ppm}\left(2 \times 10^{-7}\right)[2-4]$. The discovery of the quantum Hall effect (QHE) has initiated a large number of theoretical and experimental publications concerned with the physics of the 2DEG. Some aspects of the work concerned with the transport behaviour of a $2 \mathrm{DEG}$ that has been done during the past few years at our laboratory will be summarized in this paper.

\section{Some Essential Characteristics Concerned with the QHE}

A quasi two-dimensional electron gas is present in those systems where the electrons are confined within a narrow potential well whose width is small compared to the de Broglie wavelength of the carriers. This can be realized, for example, at the $\mathrm{Si}-\mathrm{SiO}_{2}$ or the $\mathrm{GaAs}-\mathrm{Al}_{\mathrm{x}} \mathrm{Ga}_{1-x} \mathrm{As}$ interface of a Si-MOSFET or a GaAs- $\mathrm{Al}_{\mathrm{x}} \mathrm{Ga}_{1-\mathrm{x}} \mathrm{As}$ heterostructure, respectively. In the presence of a strong magnetic field the motion parallel to the interface, i.e. $x-y$ plane, becomes quantized because the electrons are now forced to move in cyclotron orbits. This leads to an energy spectrum consisting of $\mathrm{n}$ equidistant Landau levels:

$E_{\mathrm{j}, \mathrm{n}}=E_{\mathrm{j}}+\left(\mathrm{n}+\frac{1}{2}\right) \hbar \omega_{\mathrm{c}} ; \mathrm{n}=0,1,2, \ldots$

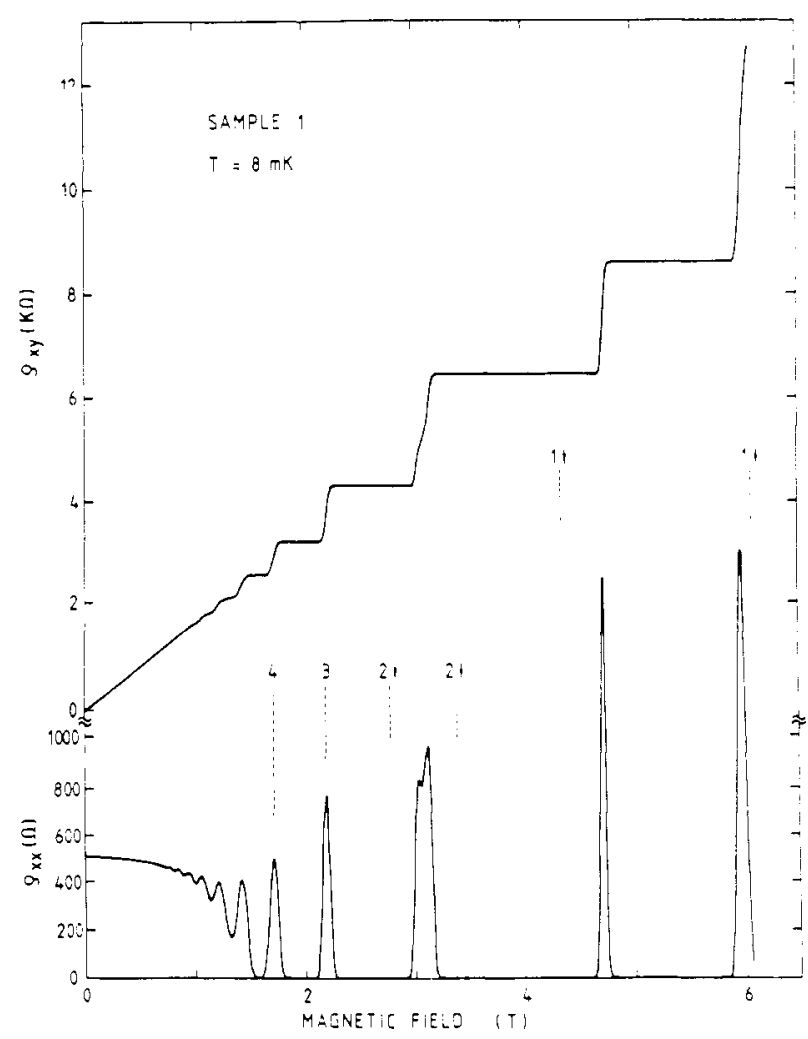

Fig. 1. Resistivity $\varrho_{x x}$ and Hall resistivity $\varrho_{x y}$ as a function of magnetic field at $T=8 \mathrm{mK}$ for a $\mathrm{GaAs}-\mathrm{Al}_{\mathrm{x}} \mathrm{Ga}_{1-\mathrm{x}}$ As heterostructure 


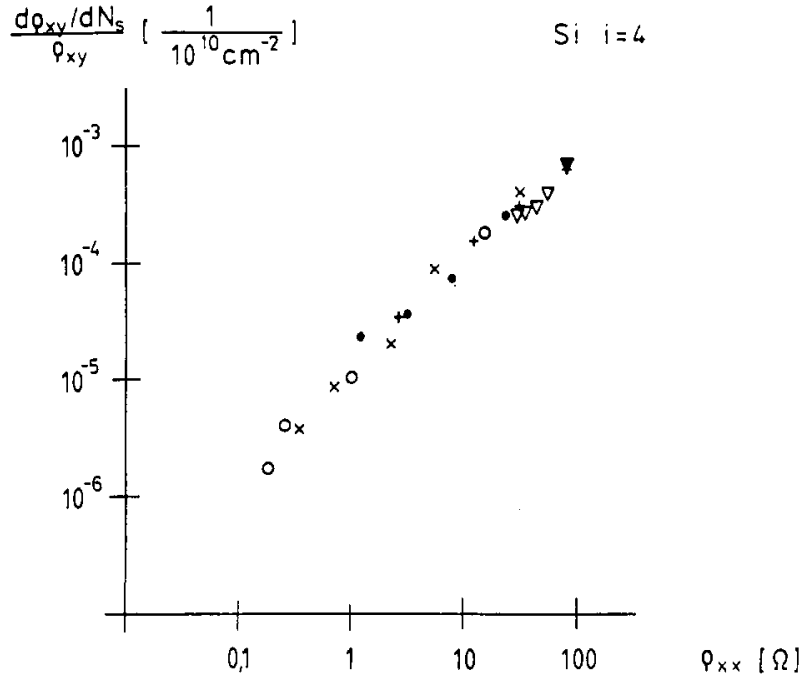

Fig. 2. Slope of the resistance $\mathrm{d} \varrho_{x y} / \mathrm{d} V_{\mathrm{g}} \sim \mathrm{d} \varrho_{x y} / \mathrm{dn}_{\mathrm{s}}$ at the centre of the Hall plateaux as a function of the minimal resistivity $g_{x x}$. The magnetic field has been varied between $11 \mathrm{~T}$ and $13 \mathrm{~T}$, the temperature between $1.5 \mathrm{~K}$ and $4.2 \mathrm{~K}$ and the substrate bias voltage between $-9 \mathrm{~V}$ and zero

and the number of states per unit area $\mathrm{N}_{L}$ within each Landau level is $\mathrm{N}_{\mathrm{L}}=e B / h$. Therefore, if exactly an integer number $i$ of Landau levels is occupied $\left(\mathrm{n}_{\mathrm{s}}=\mathrm{i} \cdot \mathrm{N}_{\mathrm{L}}\right)$ the Hall resistance $\varrho_{x y}$ becomes quantized:

$\varrho_{x y}=\frac{B}{e \mathrm{n}_{\mathrm{s}}}=\frac{h}{e^{2} \mathrm{i}}$.

As shown in Fig. 1, one finds experimentally that $\varrho_{x y}$ is no longer a monotonic function but there are certain plateau regions where the Hall resistance becomes constant. Moreover, the value of $\varrho_{x y}$ is given by (2) provided that the magnetoresistance $\varrho_{x y}$ is zero in this region.

In the meantime, both the flatness of the plateaux [3] and their absolute value [2] have been verified within an uncertainty of $\Delta \varrho_{x y} / \varrho_{x y} \leqslant 0.06 \mathrm{ppm}$ but, as indicated before, there is a strong correlation between the flatness of the plateaux and $\varrho_{x x}$ (Fig. 2).

\section{Current Distribution Within a Hall Sample}

In order to increase the resolution of high-precision measurements of the quantized Hall resistance it would be desirable to increase the applied current. However, measurements of the resistivity in the minima of the Shubnikov-de Haas oscillations demonstrate that at temperatures of liquid helium an instability in the current-voltage characteristic occurs if the current is increased above a certain value [5] (Fig. 3).

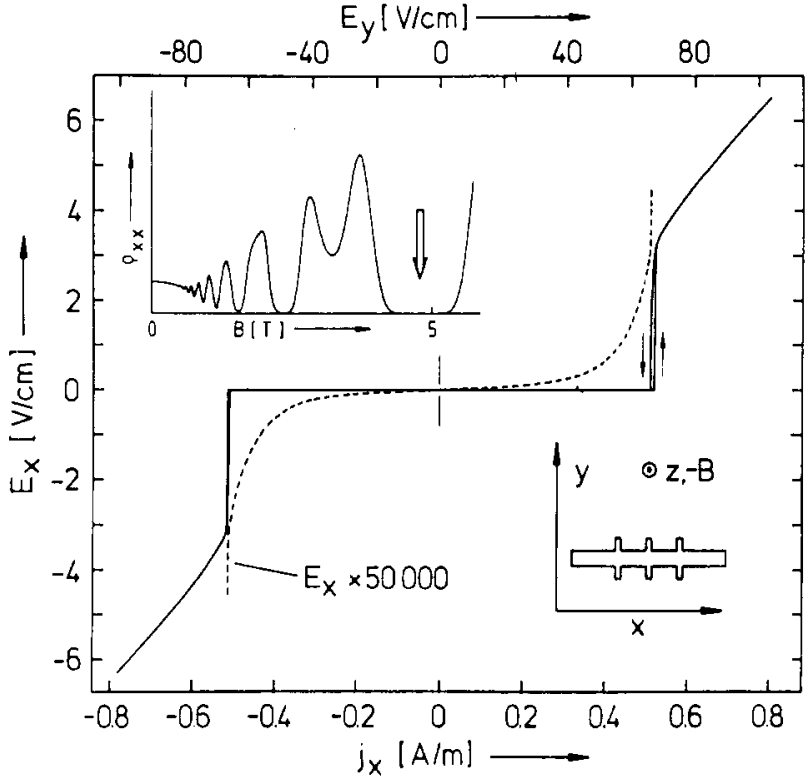

Fig. 3. Current-voltage characteristic of a $\mathrm{GaAs}-\mathrm{Al}_{\mathrm{x}} \mathrm{Ga}_{1-x} \mathrm{As}$ heterostructure at $B=4.7 \mathrm{~T}$ and $T=1.4 \mathrm{~K}$ The device geometry and the Shubnikov-de Haas oscillations are shown in the inserts. For the dotted line the $E_{x}$-axis is magnified by a factor of 50,000
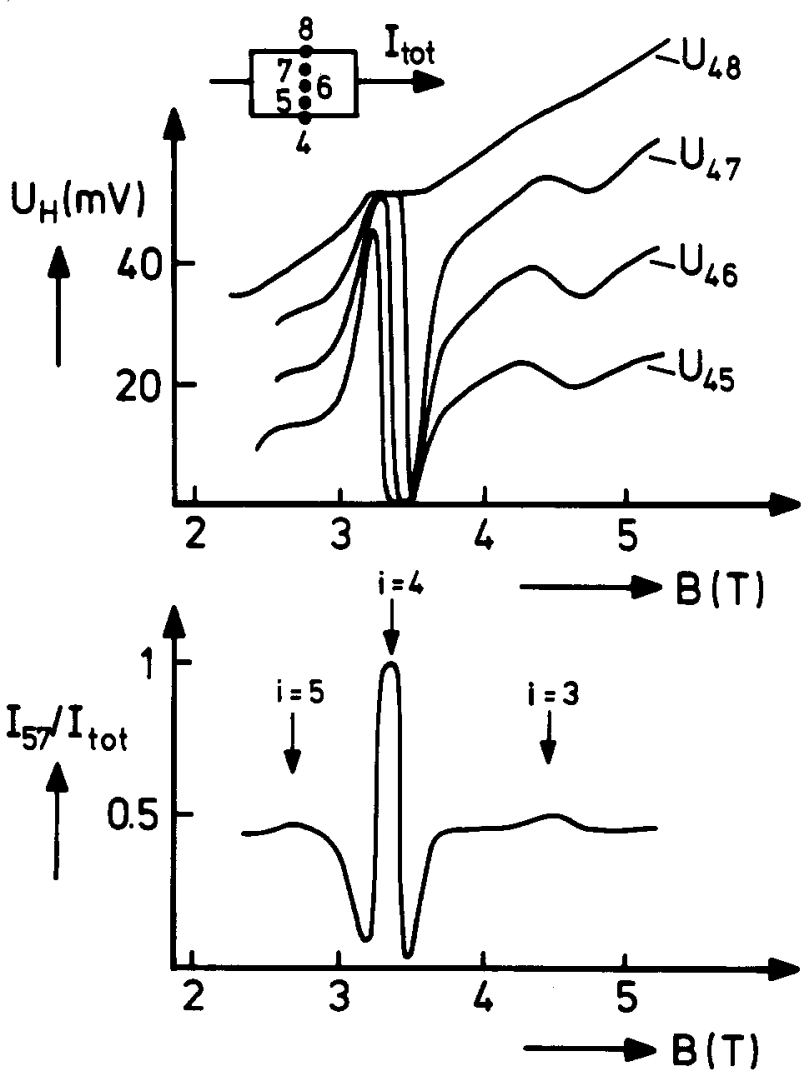

Fig. 4. Hall voltages $U_{45}, U_{46}, U_{47}$ and $U_{48}$ as a function of the magnetic field. The lower curve shows the fraction of the current flowing between the potential probes 5 and 7 at magnetic fields close to the filling factor $i=4$ 
Since the drift velocity $v_{\mathrm{D}}=E_{y} / B$ at the critical current is of the same order of magnitude as the sound velocity $v_{\mathrm{s}} \cong 2.5 \times 10^{3} \mathrm{~m} / \mathrm{s}$, phonon-stimulated effects have been proposed as an interpretation of the breakdown $[6,7,10]$. Nevertheless, the problems concerned with the non-equilibrium behaviour have to be considered as mainly unsolved because up to now no definite answer has been found upon the question of Hall potential distribution within a real sample.

Ebert et al. [11] investigated the current distribution on GaAs- $\mathrm{Al}_{x} \mathrm{Ga}_{1-x}$ As heterostructures with standard Hall geometry but additional ohmic contacts across the width of the sample, as shown in the insert of Fig. 4. Special care was taken to avoid the Corbino effect on the inner contacts by measuring at finite temperatures $T>3.3 \mathrm{~K}$ and magnetic fields smaller than $B=6 \mathrm{~T}$ where the magnetoconductivity was typically larger than $\sigma_{x x}=10^{-8} \Omega^{-1}$.

Figure 4 indicates that for those magnetic fields where the system is not within the quantum Hall regime the Hall voltages $U_{4 \mathrm{x}}$ are nearly proportional to the distance between the different pairs of Hall probes, as one would expect for a homogeneous current distribution. However, at integer filling factors and especially in the QHE regime this doesn't hold any longer. The general behaviour can be studied, for example, by analyzing the Hall voltage between the Hall probes 4 and 6 relative to $V(4-8)$. When passing through the plateau region the measured voltage $V(4-6)$ is drastically enhanced up to a magnetic field $B$ close to the center of the plateau and then drops down when $B$ is further increased.

In the lower part of Fig. 4 the current $I(5-7)$ flowing between the potential probes 5 and 7 , normalized to the total current $I(4-8)$, is plotted. More than $90 \%$ of the total current is flowing in the inner part of the device (between the potential probes 5 and 6) in the magnetic field range corresponding to a variation of the filling factor from $\mathrm{i}=3.92$ to $\mathrm{i}=4.08$. Less than $10 \%$ of the current is flowing in this part of the sample at filling factors $\mathrm{i}=3.88$ and $\mathrm{i}=4.12$.

We believe that the inhomogeneous current distribution is due to a gradient in the carrier density across the width of the device. The main current shifts from one side of the sample to the other one within the quantum Hall plateau. The importance of a carrier density gradient in the direction of the Hall field has been demonstrated by producing a spatially-varying carrier density using a rear gate tilted relative to the plane of the two-dimensional system. Fig. 5 shows clearly that such an external gradient of the gate field across the width of the sample changes the current distribution within the device completely.

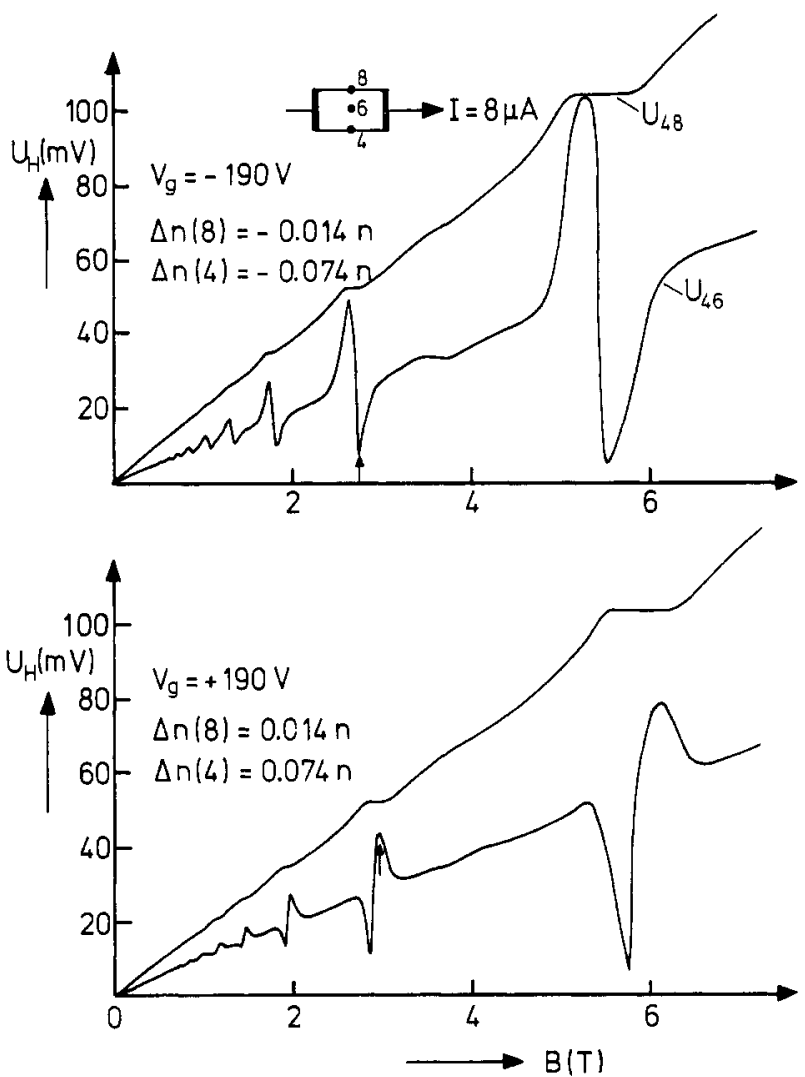

Fig. 5. Hall voltages $U_{48}$ (voltage across the entire sample) and $U_{46}$ (voltage across half of the width of the sample) at $T=3.3 \mathrm{~K}$ as a function of magnetic field. An inhomogeneous carrier density with a gradient in the direction of the Hall field has been produced by a gate voltage between the twodimensional system and a metal plate on the back of the sample

Theoretical calculations [7-9] predict that the Hall potential drop is confined within a certain distance from the boundaries of the sample, but they do not enable one to explain the experimental results. This is probably due to the fact that they do not include localization, inhomogeneities and the finite size of real samples in all directions.

\section{Density of States}

The quantization of the Hall resistivity into the integer multiple of $h / e^{2}$ indicates the presence of both localized and extended states in Landau levels. Theoretical efforts have in particular been made to calculate the shape of the density of states (DOS) [12-14].

A method to measure the DOS between the Landau levels follows from the analysis of the thermally activated resistivity. The basic idea is that at temperatures where the thermal activation of localized carriers is dominant the resistivity depends 


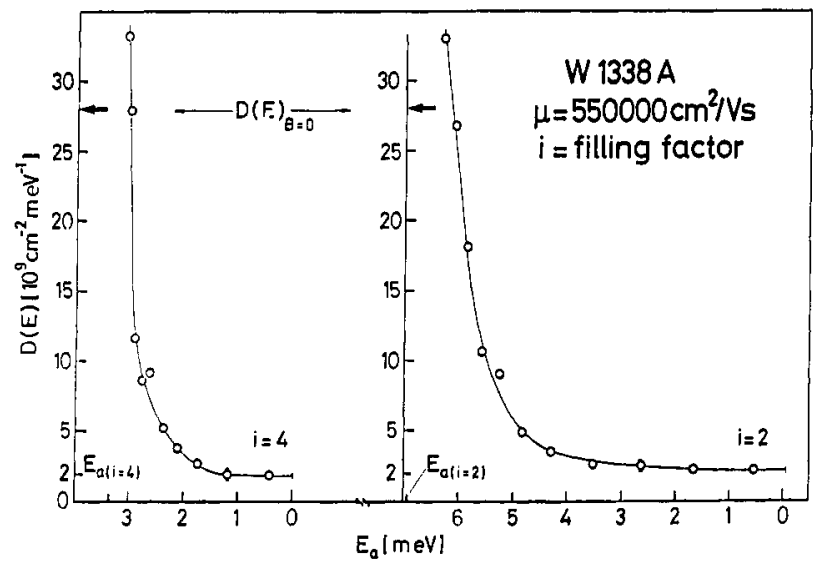

Fig. 6. The density of states within two different mobility gaps of a $\mathrm{GaAs}-\mathrm{Al}_{\mathrm{x}} \mathrm{Ga}_{1-\mathrm{x}} \mathrm{As}$ heterostructure with mobility $\mu=550,000 \mathrm{~cm}^{2}(\mathrm{Vs})^{-1}$. The centres of the Landau levels are denoted with $E_{\mathrm{a}(\mathrm{i}=2)}$ and $E_{\mathrm{a}(\mathrm{i}=4)}$

on the relative position of the Fermi energy with respect to the mobility edge $E_{\mathrm{c}}$. In a simplified equation $\varrho_{x x}$ is given by

$\varrho_{x x}=\varrho_{0} \exp \left\{-\left(E_{\mathrm{c}}-E_{\mathrm{F}}\right) / k T\right\}$.

The Fermi level $E_{\mathrm{F}}$ is changed, for example, by varying the carrier density of the $2 \mathrm{DEG}$ according to

$\Delta \mathrm{n}_{\mathrm{S}}=\int f\left(E_{\mathrm{F}_{1}}\right) D(E) \mathrm{d} E-\int f\left(E_{\mathrm{F}_{2}}\right) D(E) \mathrm{d} E$.

From this formula it becomes clear that, for small values of $\Delta n_{s}$, an average density of localized states can be computed in a good approximation.

In Fig. 6 the density of a $2 \mathrm{DEG}$ in a strong magnetic field measured on a GaAs- $\mathrm{Al}_{\mathrm{x}} \mathrm{Ga}_{1-\mathrm{x}} \mathrm{As}$ heterostructure with a mobility $\mu=550000 \mathrm{~cm}^{2}$ $(V s)^{-1}$ is plotted for filling factors $\mathrm{i}=2$ and $\mathrm{i}=4$. It turns out that the DOS does not decrease exponentially with increasing magnetic field because the minimal DOS is larger than $2 \times 10^{9} \mathrm{~cm}^{-2} \mathrm{meV}^{-1}$ and is nearly the same for filling factors $i=2$ and $i=4$. The minimal DOS increases with decreasing mobility and for a sample with a mobility of $\mu=105000$ $\mathrm{cm}^{2}(\mathrm{Vs})^{-1}$ a value of about $5 \times 10^{9} \mathrm{~cm}^{-2} \mathrm{meV}^{-1}$ has been obtained. According to our measurements the DOS is best described by a Gaussian density of states superimposed upon a constant background.

\section{Thermomagnetic Behaviour of the 2DEG}

In usual precision determinations of the quantized Hall resistance the applied current $I$ is of the order of $10 \mu \mathrm{A}$. Consequently, if one wants to determine $\varrho_{x y}=h /\left(2 e^{2}\right)$ (at the filling factor $\mathrm{i}=2$ ) within an uncertainty of $0.01 \mathrm{ppm}$ the resolution $\delta V$ of the Hall voltage has to be about $1 \mathrm{nV}$. This leads to the question whether thermoelectric effects of the 2DEG may cause any systematic error.

The Seebeck and the Nernst-Ettingshausen effects have been investigated by using a standard differential measuring technique where the thermopower $S$ is given by the ratio of the thermal voltages $V_{x x}^{\mathrm{th}}$ and $V_{x y}^{\mathrm{th}}$, respectively, and the temperature difference $\Delta_{x} T$. The temperature gradient in the direction of the long axis of the device was obtained by connecting the sample to an electric heater and to a heat sink, the temperature difference $\Delta T$ along the sample being measured with AuFe-chromel thermocouples.

As shown in Fig. 7, the diagonal component of the thermopower $S_{x x}$ oscillates as a function of magnetic field. In an earlier paper [15] it has been demonstrated that the maxima and minima in $S_{x x}$ coincide with those of the magnetoresistance of a 2 DEG. Within the plateau region of the quantum Hall effect (see insert of Fig. 7) $S_{x x}$ vanishes as well as $S_{x y}$ [15], indicating that errors due to thermal voltages will be very small at low temperatures.

Recently, it was predicted $[16,17]$ that at low temperatures the maxima of the Seebeck oscillations $S_{x x}^{\max }$ should become

$\left.S_{x x}^{\max }\right|_{\text {theory }}=-\frac{k}{e}\left(\frac{1}{\mathrm{~N}_{\mathrm{L}}+\frac{1}{2}}\right) \cdot \ln 2$

$\left(\mathrm{N}_{\mathrm{L}}\right.$ : quantum number of the Landau level)

and therefore should be independent of device parameters. Indeed, on some samples with mobilities higher than about $50000 \mathrm{~cm}^{2}(\mathrm{Vs})^{-1}, S_{x x}^{\max }$ has been found to be constant and equal to the value predicted in (5) at temperatures lower than $5 \mathrm{~K}$ [15]. On samples with lower mobilities, however, the maxima of the thermopower oscillations have been

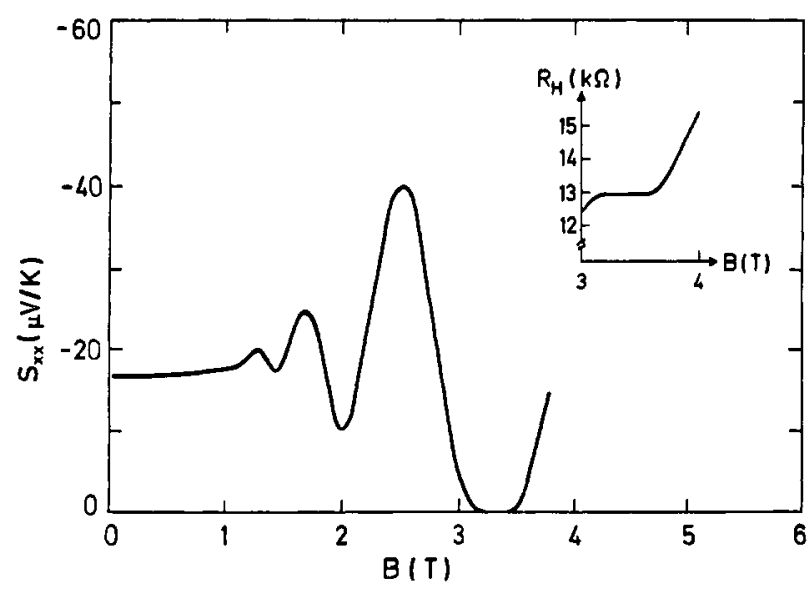

Fig. 7. Diagonal component $S_{x x}$ of the thermopower tensor in the magnetic field range $0<B<4 \mathrm{~T}$. For comparison the Hall resistance $R_{\mathrm{H}}$ between $B=3 \mathrm{~T}$ and $B=4 \mathrm{~T}$ is plotted in the insert 


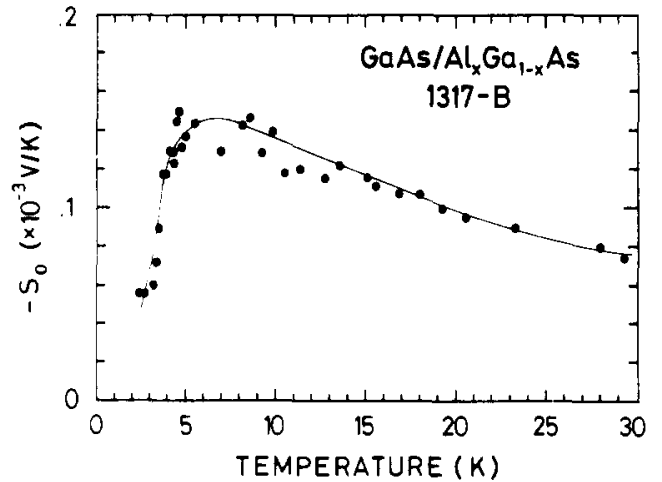

Fig. 8. Thermopower $S_{0}$ at zero magnetic field as a function of temperature. The mobility of the investigated sample was $208,000 \mathrm{~cm}^{2}(\mathrm{Vs})^{-1}$

observed to be smaller than $\left.S_{x . x}^{\max }\right|_{\text {Theory }}[15,18]$. This is due to the fact that for these devices the Landaulevel linewidth $\Gamma$ compared to $\hbar \omega_{c}$ can no longer be neglected.

So far, only the so-called diffusion part of the thermopower has been considered, but it is well known that a strong contribution to the thermopower may be caused by the phonon-drag effect. Especially, if the mean free path of the phonons becomes of the order of the dimensions of the device, i.e. several $\mathrm{mm}$, the drag of electrons due to phonons is expected to be very high.

In Fig. 8 the temperature dependence of the thermopower $S_{0}$ at zero magnetic field for a sample with a mobility of $208000 \mathrm{~cm}^{2}(\mathrm{Vs})^{-1}$ is plotted. Neither the height of the value of $S_{0}$ nor the variation of $S(T)$ can be explained by a purely electronic behaviour. Recently, Nicholas [19] published a theory about the phonon-drag effect $S^{\text {ph }}$ in twodimensional systems, based on an idea of Herring [20]. A simple estimation shows that the observed value of the thermopower $S_{0}$ is of the same order as one would expect from the theory of Nicholas, if a mean free path of the phonons limited only by the thickness of the bulk material is taken into account.

\section{Unusual Behaviour of the Magnetoresistance}

A general understanding of the transport behaviour of a $2 \mathrm{DEG}$ is still missing. On samples with high mobilities unexpected phenomena are found. Figure 9 shows the magnetoresistivity for two different applied currents $(I=10 \mu \mathrm{A}$ and $I=0.1 \mu \mathrm{A})$ on a long sample whose length-to-width ratio is about 167. Whereas the lineshape of the upper curve $(I=10 \mu \mathrm{A})$ is comparable to the results obtained in earlier experiments on shorter samples and on devices with lower mobilities, the lower curve $(I=0.1 \mu \mathrm{A})$ looks quite different. The quantum

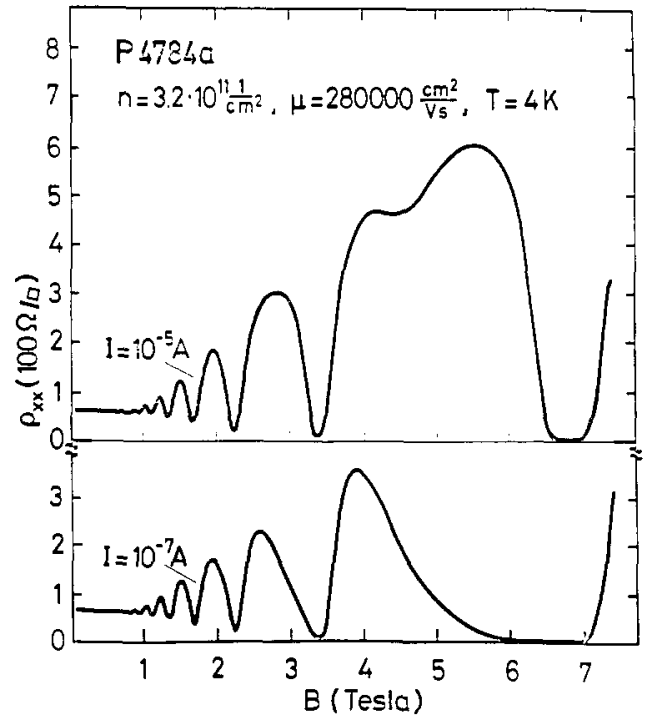

Fig. 9. Magnetoresistivity $\varrho_{x x}$ of a GaAs- $\mathrm{Al}_{x} \mathrm{Ga}_{1-x} \mathrm{As}$ heterostructure for two different applied currents $I=10^{-5} \mathrm{~A}$ and $I=10^{-7} \mathrm{~A}$

oscillations in $\varrho_{x x}$ versus $B$ appear saw-tooth shaped and the maxima in $\varrho_{x x}$ are shifted to lower magnetic fields. Moreover, in strong magnetic fields where in the upper curve the onset of spin-splitting is visible, in the lower curve one spin-level seems to have totally vanished.

The observed phenomenon is probably due to a more general mechanism because similar lineshapes have also been observed on Si-MOSFETs [21], but up to now we have no definite explanation for this effect. Recently, Zheng et al. [22] reported similar results as they reduced the width of their samples. They speculated that in samples with high mobilities any characteristic length becomes dominant and therefore the transition to a quasi one-dimensional behaviour will be observed when the size of the device is reduced.

\section{Electron Spin Resonance}

We have investigated the photoresistivity $\varrho_{x x}$ of a $2 \mathrm{DEG}$ in $\mathrm{GaAs}-\mathrm{Al}_{x} \mathrm{Ga}_{1-x}$ As heterostructures in high magnetic fields by illuminating the samples with microwave irradiation. This kind of measurement technique enables one to detect electron spin resonance (ESR) on about $10^{10}$ electrons within an area of approximately $5 \times 2 \mathrm{~mm}^{2}$.

In Fig. $10, \varrho_{x x}$ and the change in $\varrho_{x x}, \Delta \varrho_{x x}$, are plotted as a function of magnetic field. Most of the photosignal $\Delta \underline{Q}_{x x}$ arises from the heating of the electron gas by the irradiation which is not sensitive to the microwave frequency $v$ and is assumed to be a bolometric background signal. In addition, a sharp 


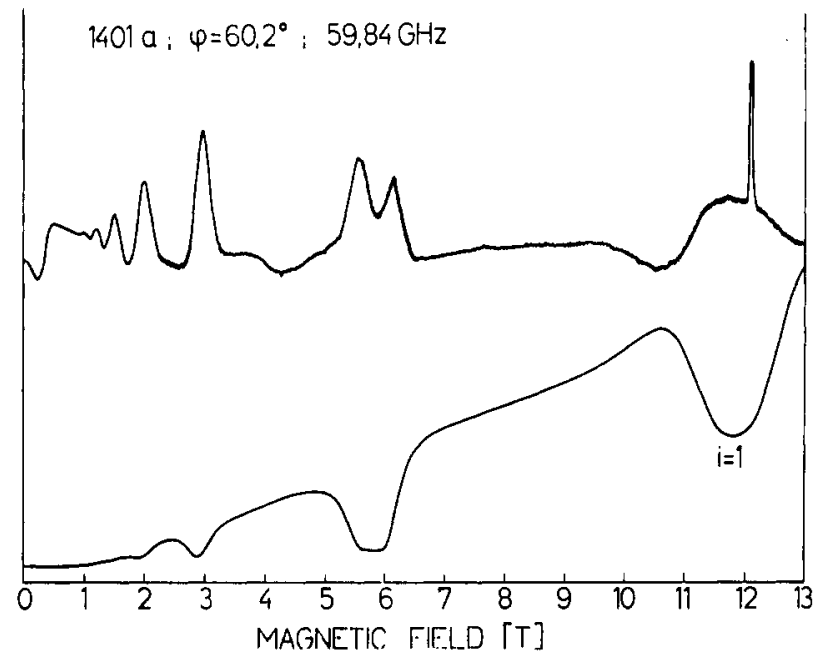

Fig. 10. Experimental data for the resistivity $\varrho_{x . x}$ and the photoresponse $\Delta \varrho_{x x}$ as a function of magnetic field

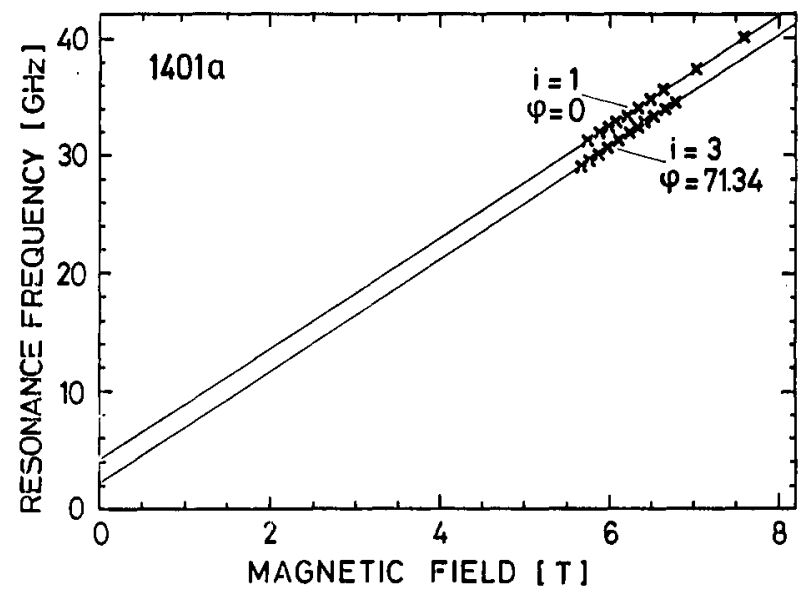

Fig. 11. Resonance frequencies $v$ as a function of magnetic field $B$ for two different filling factors $\mathrm{i}=1$ and $\mathrm{i}=3$

resonance signal occurs at a magnetic field position $B$ where the Fermi level is located in the energy gap between spin-split Landau levels as expected for ESR.

The observed resonances attributed to ESR obey to good accuracy a linear dependence of $v$ on $B$ $\left(v=v_{0}+\alpha B\right)$ (Fig. 11). As shown by Stein et al. [23], this behaviour holds also at lower magnetic fields. From the slope $\alpha$, which is proportional to the g-factor, we derived $g \approx 0.3$ depending on the carrier density of the sample and the Landau level involved.

A linear extrapolation to $B=0$ leads to a zerofield spin-splitting. This can be understood as a consequence of the lack of inversion symmetry in the triangular potential well confining the $2 \mathrm{DEG}$. Attempts have also been made to explain the measurements by a non-linear fit without zero-field splitting [24] but up to now the experimental data do not allow any reliable statement to exclude the one or the other theoretical model.

\section{References}

1. K. v. Klitzing, G. Dorda, M. Pepper: Phys. Rev. Lett. 45, 494 (1980)

2. L. Bliek, E. Braun, H. J. Engelmann, H. Leontiew, F. Melchert, W. Schlapp, B. Stahl, P. Warnecke, G. Weimann: PTB-Mitt. 93, 21 (1983)

3. G. Ebert, T. Herzog, H. Obloh, B. Tausendfreund: PTBMitt. 93, 293 (1983)

4. M. E. Cage, R. F. Dziuba, B. F. Field, C. F. Lavine, R. J. Wagner: In: Precision Measurements and Fundamental Constants II, B. N. Taylor, W. D. Phillips (eds.), Natl. Bur. Stand. (U.S.), Spec. Publ. 617 (U.S. Government Printing Office, Washington D.C., 1984), p. 539

5. G. Ebert, K. v. Klitzing, K. Ploog, G. Weimann: J. Phys. C 16, 5441 (1983)

6. P. Streda, K. v. Klitzing: J. Phys. C 17, L483 (1984)

7. O. Heinonen, P. L. Taylor, S. M. Girvin: Phys. Rev. B 30, $3016(1984)$

8. A. H. MacDonald, T. M. Rice, W. F. Brinkman: Phys. Rev. B 28, 3648 (1983)

9. J. Riess: J. Phys. C17, L 849 (1984)

10. L. Smrcka: J. Phys. C 18, 2897 (1985)

11. G. Ebert, K. v. Klitzing, G. Weimann: J. Phys. C18, L257 (1985)

12. L. Schweitzer, B. Kramer, A. Mackinnon: J. Phys. C17, $4111(1984)$

13. F. Wegner: Z. Phys. B - Condensed Matter 51, 279 (1983)

14. H. Aoki: J. Phys. C 10, 2583 (1977); 11, 3823 (1978); $17,1875(1984)$

15. H. Obloh, K. v. Klitzing, K. Ploog: Surf, Sci. 142, 236 (1984)

16. S. P. Zelenin, A. S. Kodrat'ev, A. E. Kuchma: Sov. Phys.Semicond. 16, 355 (1982)

17. S. M. Girvin, M. Jonson: J. Phys. C 15, L1147 (1982)

18. H. Obloh, K. v. Klitzing, K. Ploog, G. Weimann: (to be published in Surf. Sci.)

19. R. J. Nicholas: J. Phys. C 18, L695 (1985)

20. C. Herring: Phys. Rev. 111, 36 (1958)

21. K. v. Klitzing, G. Ebert, N. Kleinmichel, H. Obloh, G. Dorda, G. Weimann: Proc. 17th Int. Conf. Phys. Semiconductors, San Francisco 1984, J. D. Chadi, W. A. Harrison (eds.) (Springer Verlag, New York, Berlin, Heidelberg 1985), p. 271

22. H. Z. Zheng, K. K. Choi, D. C. Tsui, G. Weimann: Phys. Rev. Lett. 55, 1144 (1985)

23. D. Stein, K. v. Klitzing, G. Weimann: Phys. Rev. Lett. 51, 130 (1983)

24. G. Lommer, F. Malcher, U. Rössler: (to be published in Phys. Rev.) 